\title{
OPTIMIZACIÓN DE LA TÉCNICA DE VOLTAMPEROMETRÍA DE ADSORCIÓN (AdSV) EN LA DETERMINACIÓN DE AMARANTH SOBRE ELECTRODOS SERIGRAFIADOS DE CARBONO. EFECTO DE SURFACTANTES EN LA SENSIBILIDAD
}

\author{
Karen Liceth Bolaños ${ }^{\mathrm{a}}$, Edgar Nagles ${ }^{\mathrm{ab} *}$, Verónica Arancibia ${ }^{\mathrm{b}}$, Manuel Otiniano, \\ Yaritza Leiva ${ }^{a}$, Adrián Mariño ${ }^{a}$, Laura Scarpetta ${ }^{a}$.
}

\begin{abstract}
RESUMEN
Un sensible y simple método electroanalítico es optimizado para la determinación de amaranth por AdSV sobre un electrodo serigrafiado de carbono (Dropsens DRP-154). El amaranth (E123) es depositado por adsorción sobre la superficie del electrodo y el pico de corriente es medido por onda cuadrada (SWV). El electrodo se caracterizó por voltamperometría cíclica $(\mathrm{CV})$ en presencia del surfactante catiónico bromuro de cetilpiridina (CPB) donde el E123 es oxidado a $0,62 \mathrm{~V}$ en un proceso controlado por difusión, aumentando en $100 \%$ el pico de corriente en presencia de CPB. Las variables experimentales como $\mathrm{pH}$, concentración de surfactante $\left(\mathrm{C}_{\mathrm{CPB}}\right)$, tiempo de adsorción (tads) y potencial de adsorción $\left(\mathrm{E}_{\mathrm{ads}}\right)$, así como también las potenciales interferencias, fueron estudiadas. Bajo las óptimas condiciones ( $\left.\mathrm{pH} 2,0 ; \mathrm{C}_{\mathrm{CPB}} 1,0 \times 10^{-5} \mathrm{molL}^{-1} ; \mathrm{t}_{\mathrm{acc}} 90 \mathrm{~s} ; \mathrm{E}_{\mathrm{acc}} 0,10 \mathrm{~V}\right)$ el pico de la corriente es proporcional a la concentración de E123 entre 0,10 to $2,0 \times 10^{-6} \mathrm{molL}^{-1}$, con un límite de detección de $0,18 \times 10^{-7} \mathrm{molL}^{-1}$. La desviación estándar relativa para una solución conteniendo $1,0 \times 10^{-5} \mathrm{molL}^{-1}$ de E123 fue de $1,0 \%$ para siete medidas iguales. El método fue validado con una muestra de agua dopada con E123. Finalmente el método fue aplicado en la determinación de amaranth en muestras de sodas.

Palabras clave: Amaranth, voltamperometría de adsorción; bromuro de cetilpiridina.

\section{OPTIMIZING ADSORPTION VOLTAMMETRIC TECHNIQUE (AdSV) IN DETERMINING OF AMARANTH ON CARBON PRINTED ELECTRODES. EFFECT OF SURFACTANTS ON SENSITIVITY}

\begin{abstract}
A simple and sensitive electroanalytical method is optimized by determining amaranth(E123) by AdSV on a screen printed carbon electrode (Dropsens DRP-154). E123 is deposited by adsorption on the electrode surface and the current peak measured by square wave (SWV). The electrode is characterized by cyclic voltammetry (CV) in the presence of cationic surfactant cetylpyridinium bromide (CPB) where E123 is oxidized to $0.62 \mathrm{~V}$ by diffusion, increasing near $100 \%$ the peak current in the presence of $\mathrm{CPB}$. The experimental

\footnotetext{
${ }^{*}$ Universidad de la Amazonia, Facultad de Ciencias Básicas, Programa de Química, Florencia, Colombia.ernagles@uc.cl, e.nagles@udla.edu.co

b Facultad de Química, Pontificia Universidad Católica de Chile, Santiago, Chile, E-mail:

c Facultad de Química e Ingeniería, Universidad Nacional Mayor de San Marcos, Lima, Perú.
} 
variables such as $\mathrm{pH}$, concentration of surfactant $\left(\mathrm{C}_{\mathrm{CPB}}\right)$ adsorption time $\left(\mathrm{T}_{\mathrm{ads}}\right)$ and adsorption potential $\left(\mathrm{E}_{\mathrm{ads}}\right)$, as well as potential interference, were studied. Under optimal conditions $(\mathrm{pH}$ 2.0; $\mathrm{C}_{\mathrm{CPB}} 1 . \mathrm{x} 10^{-5} \mathrm{molL}^{-1} ; \mathrm{t}_{\mathrm{ads}} 90 \mathrm{~s} ; \mathrm{E}_{\text {ads }} 0.10 \mathrm{~V}$ ) the peak current is proportional to the concentration of E123 between 0.10 to $2.0 \times 10^{-6} \mathrm{molL}^{-1}$, with a detection limit of $0.18 \times 10^{-7}$ molL $\mathrm{L}^{-1}$. The relative standard deviation for a solution containing $1.0 \times 10^{-5} \mathrm{molL}^{-1}$ of E123 was $1.0 \%$ for seven equal measures. The method was validated with a water sample doped with E123. Finally the method was applied in the determination of amaranth in samples of sodas.

Key words: Amaranth, adsorption voltammetric, cetylpiridinium bromide.

\section{INTRODUCCIÓN}

En los últimos años la industria de alimentos ha incrementado el uso de los colorantes sintéticos debido a sus propiedades, tales como: bajo costo, estabilidad a la luz, al pH y al oxígeno ${ }^{1}$. Esto les permite tener una mayor aplicación comparada con los colorantes naturales. El amaranth (figura 1), cuyo nombre IUPAC es trisodio 2-hidroxi-1-(4-sulfonato1-naftilazo)naftaleno-3-6-disulfonato ${ }^{2}$. También llamado rojo ácido 27, es un colorante azoico conocido comúnmente por su aplicación en la industria textil y actualmente como un agente colorante en alimentos envasados. Por otro lado, en los últimos cinco años se ha determinado que su consumo frecuente produce diversos tipos de patologías, tales como: alergias, problemas respiratorios, nauseas, diarreas, tumores y defectos desde el nacimiento ${ }^{3}$, por tal motivo se prohíbe o restringe su uso en algunos países.

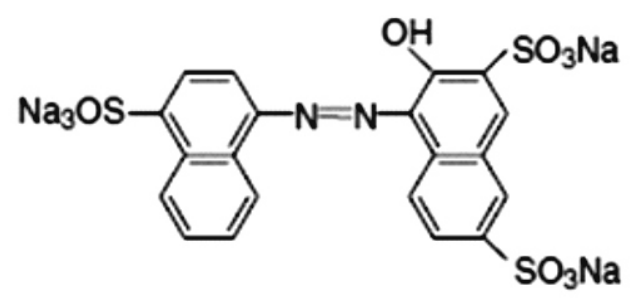

Figura 1. Estructura molecular del amaranth.

De ahí la importancia del desarrollo de nuevas metodologías que permitan su detección. Algunas de las técnicas más comunes que se emplean para su detección son la espectrofotometría ${ }^{4}$, electroforesis capilar ${ }^{5}$ y cromatografía líquida de alta eficiencia (HPLC) ${ }^{6}$, con límites de detección (LOD) de $2,15 \times 10^{-8}, 4,9 \times 10^{-6}$ y $6,6 \times 10^{-5} \mathrm{molL}^{-1}$, respectivamente. Una desventaja de usar alguna de estas técnicas es su elevado costo operacional e instrumental. Las técnicas electroanalíticas como la voltamperometría de adsorción, son muy sensibles y tienen un bajo costo operacional e instrumental siendo una alternativa muy útil para la cuantificación de amaranth?

La cuantificación y propiedades electroquímicas del amaranth han sido estudiadas sobre diferentes tipos de electrodos: pasta de carbono ${ }^{8}$ pasta de carbono modificado con nanotubos de carbono multicapa ${ }^{9}$, oro modificado con nanotubos de carbono multicapa ${ }^{10}$ y pasta de carbono modificado con fibras de $\mathrm{Al}_{2} \mathrm{O}_{3}{ }^{11}$; con límites de detección (LOD) de 5,0 x 10 $0^{-9}, 3,5 \mathrm{x}$ $10^{-9}, 6,8 \times 10^{-8}, 7,5 \times 10^{-10} \mathrm{molL}^{-1}$, respectivamente. Por otro lado, el uso de surfactantes puede 
mejorar la señal con el aumento de amaranth sobre la superficie del electrodo, donde M. Panduranga et al., usaron bromuro de cetil-trimetilamonio (CTAB) ${ }^{12}$. Además, se puede combinar el uso de surfactantes con la Voltamperometría de adsorción (AdSV). Esta técnica se basa en una previa acumulación del analito por adsorcíon y la posterior oxidación o reducción del analito depositado realizando el barrido con alguna técnica moderna como voltamperometría de pulso diferencial (DPV) o voltamperometría de onda cuadrada (SWV). Estas dos etapas aportan la gran sensibilidad a la técnica. El analito puede acumularse en la superficie del electrodo de acuerdo a diversos mecanismos ${ }^{13}$.

En este trabajo se investigó las propiedades electroanalíticas del amaranth sobre electrodos serigrafiados de carbono en presencia del surfactante CPB y se optimizó los parámetros ( $\mathrm{pH}$, $\left.\mathrm{C}_{\mathrm{CPB}}, \mathrm{E}_{\mathrm{ads}} \mathrm{y}_{\mathrm{ads}}\right)$ para la cuantificación de amaranth. El método propuesto se usó en la cuantificación de amaranth en muestras reales.

\section{Reactivos}

\section{PARTE EXPERIMENTAL}

El agua usada para lavar y preparar disoluciones fue agua desionizada $18 \mathrm{M} \Omega \mathrm{cm}$ de un sistema milliQ. o agua destilada calidad HPLC (Merck). Las disoluciones patrones con 1,65 $\mathrm{x} 10^{-3}$ y $1,65 \times 10^{-4}$ molL-1 de amaranth fueron preparadas a partir de reactivo puro de AldrichSigmad y fue preparado en agua. Las disoluciones de tampones de fosfato fueron preparadas a partir de ácido fosfórico y ajustando el $\mathrm{pH}$ requerido con disolución de $\mathrm{NaOH} 2,0 \mathrm{~mol} \mathrm{~L}^{-1} \mathrm{y}$ diluyendo 10 veces con agua.

\section{Instrumentos y equipos}

Para el desarrollo de las medidas voltamperométricas se utilizó un analizador voltamétrico BAS CV50W y una VA Computrace Metrohm, para la adquisición de datos. La celda consta de un sistema de tres electrodos: un electrodo de trabajo (serigrafiado de carbono Dropsens DRP-154), un electrodo de referencia de $\mathrm{Ag} / \mathrm{AgCl} / \mathrm{KCl} 3 \mathrm{~mol} \mathrm{~L}^{-1}$, un alambre de platino como electrodo auxiliar y barra para agitación. En la preparación de los tampones se utilizó un peachímetro Orion 430 con electrodo de membrana de vidrio.

\section{Procedimiento}

En la celda electroquímica se agregó 9,0 mL de agua desionizada $18 \mathrm{M} \Omega \mathrm{cm}$ y 1,0 mL de tampón; no es necesario desgasar. Se trazó el blanco. Posteriormente se agregó el amaranth entre $1,5 \times 10^{-7}$ y $1,65 \times 10^{-6} \mathrm{molL}^{-1}$ para AdSV, y $1,65 \times 10^{-3} \mathrm{molL}^{-1}$ para voltamperometría cíclica y se trazó el respectivo voltamperograma de amaranth. En las curvas de calibrado se adicionó la misma cantidad de agua desionizada, y tampón.

\section{RESULTADOS Y DISCUSIONES}

\section{Respuesta electroquímica de amaranth sobre un electrodo serigrafiado de carbono en la presencia de CPB.}

La respuesta de amaranth sobre un electrodo de carbono serigrafiado de carbono es mostrado en la figura 2 . Con $1,65 \times 10^{-4} \mathrm{~mol} \mathrm{~L}^{-1}$ de amaranth curva negra y amaranth con $2,47 \times 10^{-5} \mathrm{~mol}$ L-1 de CPB. En buffer acetato $\mathrm{pH} 2,0$. Velocidad de barrido $(-) 50 \mathrm{mV} \mathrm{s}^{-1}$. Se observa claramente un proceso irreversible con una señal de oxidación de amaranth a $0,62 \mathrm{~V}$ (curva negra), cuando pequeñas cantidades de CPB son adicionadas se observa un aumento de casi un $100 \%$ de la señal sin CPB y aparece en -0,49 la reducción de la señal de oxidación haciendo una poco menos irreversible es sistema pero con un $\Delta \mathrm{E}$ muy alto de $0,13 \mathrm{~V}$ que indica un sistema irreversible. 
La oxidación de la señal puede ser atribuida al grupo $\mathrm{OH}$ el cual no se encuentra disociado a un $\mathrm{pH}$ de 2,0. Por otro lado, el aumento de la corriente indica que más cantidad de amaranth se está oxidando en la superficie del electrodo y esto puede ser causado por la difusión del CPB a la superficie del electrodo y al mismo tiempo ocurre una interacción por carga entre la carga positiva del CPB y las cargas negativas de los grupos sulfónicos del amaranth.

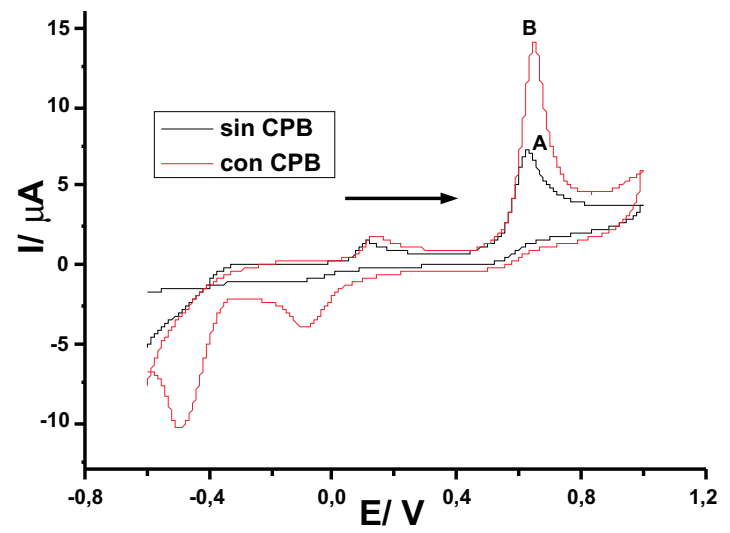

Figura 2: Voltamperogramas cíclicos de amarnth. A) sin CPB, B) con CPB; condiciones: amaranth: $1.65 \times 10^{-4} \mathrm{molL}^{-1}$; CPB: $2.47 \times 10^{-5} \mathrm{molL}^{-1}$; $\mathrm{pH}: 2.0 ;-: 100 \mathrm{mV} \mathrm{s}^{-1}$.

La electroquímica respuesta del amaranth en presencia de CPB puede ser usada para mejorar la sensibilidad de la metolodología

\section{Estudio en función del pH.}

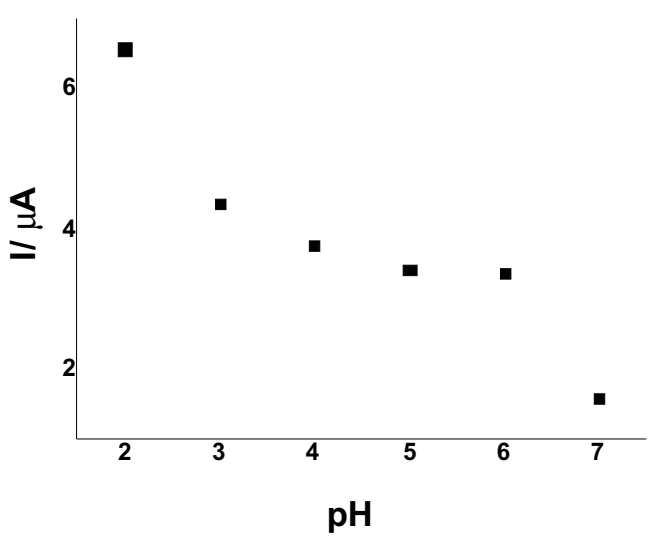

Figura 3: Efecto del $\mathrm{pH}$ en la corriente de pico del amaranth: $1,65 \times 10^{-4} \mathrm{molL}^{-1}$; velocidad de barrido $(-)$ de $50 \mathrm{mV} \mathrm{s}^{-1}$ 
Uno de los parámetros más importantes es el pH de la disolución. La disociación del grupo OH del amaranth responsable de la señal de oxidación ocurre a valores de $\mathrm{pH}$ no tan ácidos. Este estudio se realizó por $(\mathrm{CV})$ con $1,65 \times 10^{-4} \mathrm{molL}^{-1}$ de amaranth, y se varió el $\mathrm{pH}$ de la disolución entre 2,0 y 7,0 utilizando $1 \mathrm{~mL}$ de tampón fosfato de concentración $0,4 \mathrm{~mol} \mathrm{~L}^{-1}$. Se aplicó una velocidad de barrido $(-)$ de $50 \mathrm{mV} \mathrm{s}^{-1}$. Los resultados se presentan en la figura 3. Como se muestra, la mayor corriente de pico se obtuvo a $\mathrm{pH}$ 2,0 y luego a $\mathrm{pH}$ mayores decrece rápidamente. Probablemente causado por la disociación del $\mathrm{H}^{+}$del grupo $\mathrm{OH}$ a valores de $\mathrm{pH}$ menos ácidos. Este valor de $\mathrm{pH}$ fue usado en los estudios posteriores.

Estudio en función de la concentración de surfactante $\left(\mathrm{C}_{\mathrm{CPB}}\right)$.

El (CPB) cuyo nombre IUPAC es (1-hexadecylpyridinium bromide) forma micelas a concentraciones mayores de $1,0 \times 10^{-4} \mathrm{molL}^{-1}$ donde se pierde interacción con agregados en interfases. A concentraciones menores no hay micelas y su interacción con agregados en solución mejora tanto por carga como por afinidad. Para este estudio se utilizó una disolución de amaranth $1,65 \times 10^{-5} \mathrm{molL}^{-1}$ y se varió la concentración de surfactante entre $2,5 \times 10^{-6}$ y 15,0 x $10^{-6}$ molL-1 (en disolución). Los voltamperogramas fueron realizados usando onda cuadrada. Las otras condiciones fueron: $\mathrm{pH}: 2,0 ; \mathrm{t}_{\mathrm{ads}}$ (tiempo de adsorción): $60 \mathrm{~s} ; \mathrm{E}_{\text {ads }}$ (potencial de acumulación): $-0,10 \mathrm{~V}$; amplitud del escalón: $4 \mathrm{mV}$; amplitud del pulso: $25 \mathrm{mV}$ y frecuencia de $15 \mathrm{~Hz}$. Los resultados obtenidos se presentan en la figura 4
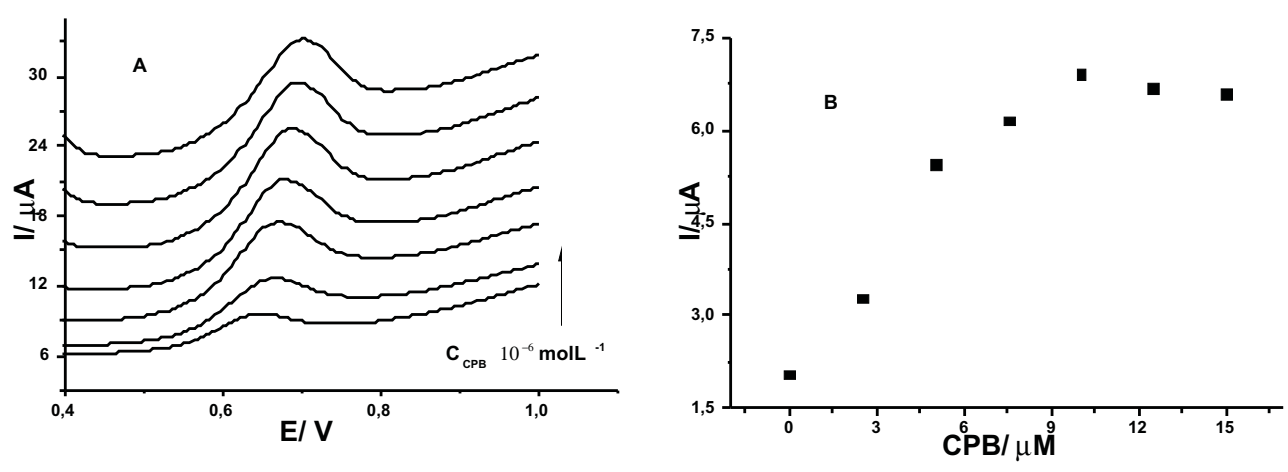

Figura 4: Efecto de la concentración de surfactante. $\left.\left(\mathrm{C}_{\mathrm{CBP}}\right) \mathrm{A}\right)$ voltamperogramas, $\left.\mathrm{B}\right)$ corriente de pico del complejo de amaranth; $1,65 \times 10^{-5} \mathrm{molL}^{-1} ; \mathrm{pH}: 2,0 ; \mathrm{t}_{\text {ads }}: 60 \mathrm{~s} ; \mathrm{E}_{\text {ads }}-0,10 \mathrm{~V}$; amplitud del escalón: $4 \mathrm{mV}$; amplitud del pulso: $25 \mathrm{mV}$ y frecuencia de $15 \mathrm{~Hz}$.

Como muestra la figura 4B, la corriente de pico aumenta con la concentración de CPB desde $2,5 \times 10^{-6} \mathrm{molL}^{-1}$ hasta $10,0 \times 10^{-6} \mathrm{molL}^{-1}$ y luego se mantiene constante. Posiblemente causado por la formación de agregados de surfactantes que saturan la superficie del electrodo. Para las medidas posteriores se eligió un valor de $10,0 \times 10^{-6} \mathrm{molL}^{-1} \mathrm{de} C P B$.

\section{Efecto de la velocidad de barrido (U)}

La figura 5 muestra el efecto de la raíz cuadrada de la velocidad de barrido $(-)^{1 / 2}$ sobre la corriente de pico de $1,65 \times 10^{-4} \mathrm{molL}^{-1}$ de amaranth entre 5 y $100 \mathrm{mV} \mathrm{s}^{-1}$. Los resultados muestran un incremento lineal de la corriente de pico entre 3,0 y $8,0(-)^{1 / 2}(\mathrm{R}=0,97)$; indicando que el proceso electroquímico es controlado por difusión. A valores mayores de $(-)^{1 / 2}$ la corriente de pico decrece indicando que la transferencia de carga no es rápida. 
Estudio en función del potencial de adsorción $\left(\mathrm{E}_{\mathrm{ads}}\right)$.

Este estudio se realizó utilizando una disolución de $1,65 \times 10^{-6} \mathrm{molL}^{-1}$ y se varió el potencial de adsorción entre 0,0 y $0,6 \mathrm{~V}$ mientras las otras condiciones permanecieron constantes: $\mathrm{pH}$ 2,0; $\mathrm{C}_{\mathrm{CPB}} 10,0 \times 10^{-6} \mathrm{molL}^{-1} ; \mathrm{t}_{\mathrm{ads}} 60 \mathrm{~s}$; Los resultados obtenidos se presentan en la figura 6. Otros parámetros iguales a figura 4 .

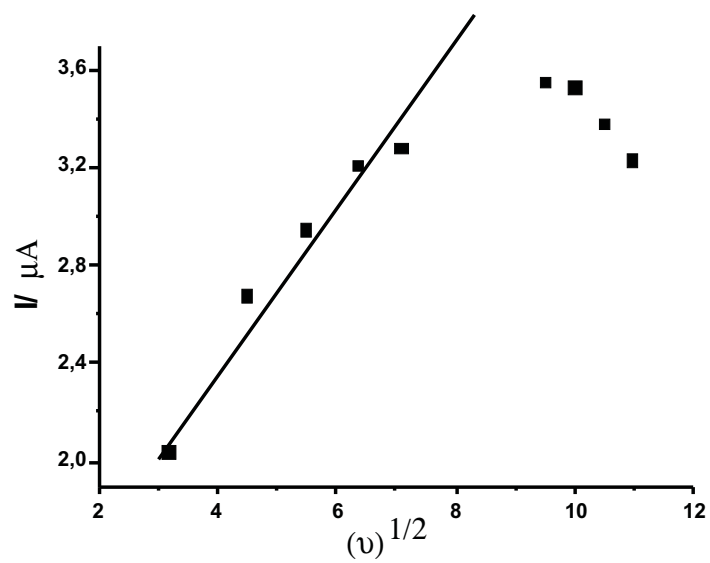

Figura 5: Efecto de la raíz de la velocidad de barrido $(-)^{1 / 2}$; $\mathrm{pH}: 2.0$; tads: $60 \mathrm{~s}$; Eads: $-0,10 \mathrm{~V}$.

Como muestra la figura 6 , la corriente de pico aumenta hasta un potencial de adsorción de 0,40 $\mathrm{V}$, para luego decrecer bruscamente. El valor de 0,40 V fue elegido para las posteriores mediciones.

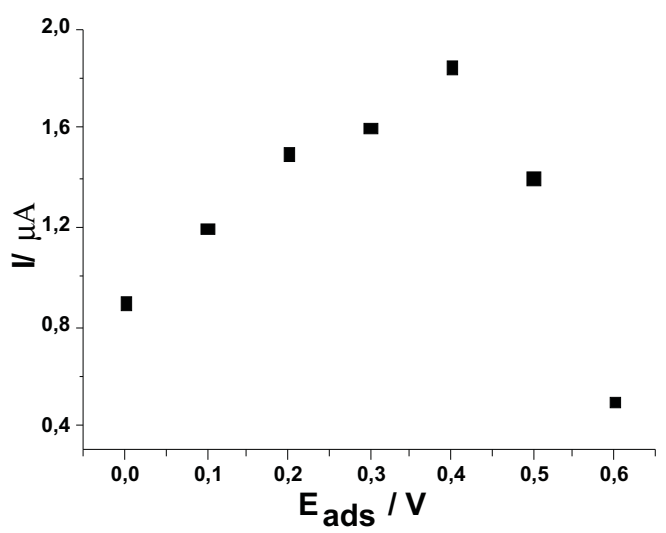

Figura 6. Efecto del potencial de adsorción $\left(\mathrm{E}_{\mathrm{ads}}\right)$ en la corriente de pico de amaranth; $1,65 \times 10^{-6} \mathrm{molL}^{-1}$; tads: $60 \mathrm{~s}$; otros parámetros iguales a figura 4 . 
Estudio en función del tiempo de acumulación (tads).

Este estudio se realizó utilizando una disolución $1,65 \times 10^{-6} \mathrm{molL}^{-1}$ de amaranth y se varió el tiempo de adsorción entre $0,0-110 \mathrm{~s}$ mientras las otras condiciones permanecieron constantes: $\mathrm{pH} 2,0 ; \mathrm{C}_{\mathrm{CPB}} 10,0 \times 10^{-6} \mathrm{molL}^{-1} ; \mathrm{E}_{\text {ads }} 0,40 \mathrm{~V}$; amplitud del paso: $10 \mathrm{mV}$; amplitud del pulso: 50 $\mathrm{mV}$ y frecuencia de $25 \mathrm{~Hz}$. Los resultados obtenidos se presentan en la figura 7.

Como muestra la figura 7, la corriente de pico aumenta con el $\mathrm{t}_{\mathrm{ads}}$ hasta $90 \mathrm{~s}$ y luego decrece ligeramente, posiblemente por la saturación de la superficie del electrodo. El $t_{\text {ads }}$ escogido fue 90 s.

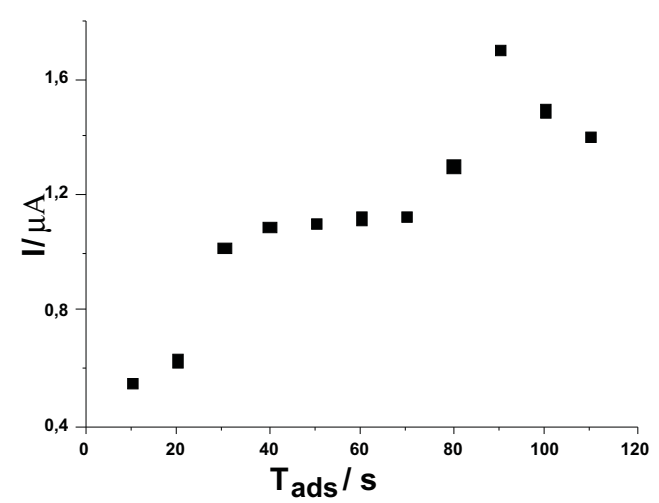

Figura 7. Efecto del tiempo del adsorción (tads) en la corriente de amaranth; 1,65 x $10^{-6}$ molL ${ }^{-1}$ pH: 2,0; $\mathrm{C}_{\mathrm{CPB}}: 10,0 \times 10^{-6} \mathrm{molL}^{-1}$; Eads $0,40 \mathrm{~V}$; otros parámetros iguales a figura 4.

\section{Parámetros de la técnica en la etapa de barrido.}

Al igual que en los estudios anteriores se eligió un electrodo serigrafiado de carbono de $4 \mathrm{~mm}$ (figura 8), la oda cuadrada con una frecuencia de $15 \mathrm{~Hz}$, un potencial del escalón de $4 \mathrm{mV}$ y una amplitud del pulso de $25 \mathrm{mV}$. A otros valores diferentes se pierde la forma del pico de oxidación.

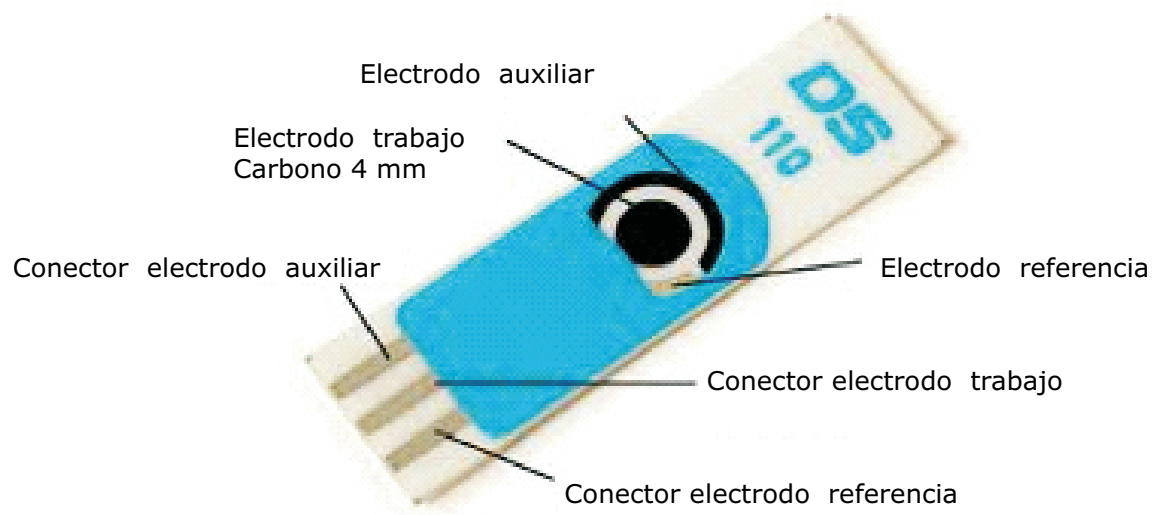

Figura 8. Esquema del electrodo serigrafiado de carbono DROPSENS. 


\section{Construcción de la curva de calibrado.}

La figura 9 muestra los voltamperogramas de adsorción en función de la concentración de amaranth; fueron obtenidos en las siguientes condiciones experimentales: $\mathrm{pH} 2,0(100 \mu \mathrm{L}$ de tampón fosfato $\left.0,4 \mathrm{~mol} \mathrm{~L}^{-1}\right) ; \mathrm{C}_{\mathrm{CPB}}: 10 \times 10^{-6} \mathrm{~mol} \mathrm{~L}^{-1} ; \mathrm{t}_{\mathrm{ads}}: 90 \mathrm{~s} \mathrm{y}_{\mathrm{ads}}: 0,40 \mathrm{~V}$.

La curva de calibrado de amaranth tiene un rango de linealidad de $0,10-2,0 \times 10^{-6} \mathrm{molL}^{-1}$. La precisión expresada como la desviación estándar relativa (R.S.D) fue de 1,0\% para siete medidas consecutivas de la misma muestra conteniendo $1,0 \times 10^{-5} \mathrm{~mol} \mathrm{~L}^{-1}$ de amaranth. El límite de detección (LD) según el método Milller and Miller ${ }^{14}$ fue de $0,18 \times 10^{-7} \pm 0,2$ molL $^{-1}$.
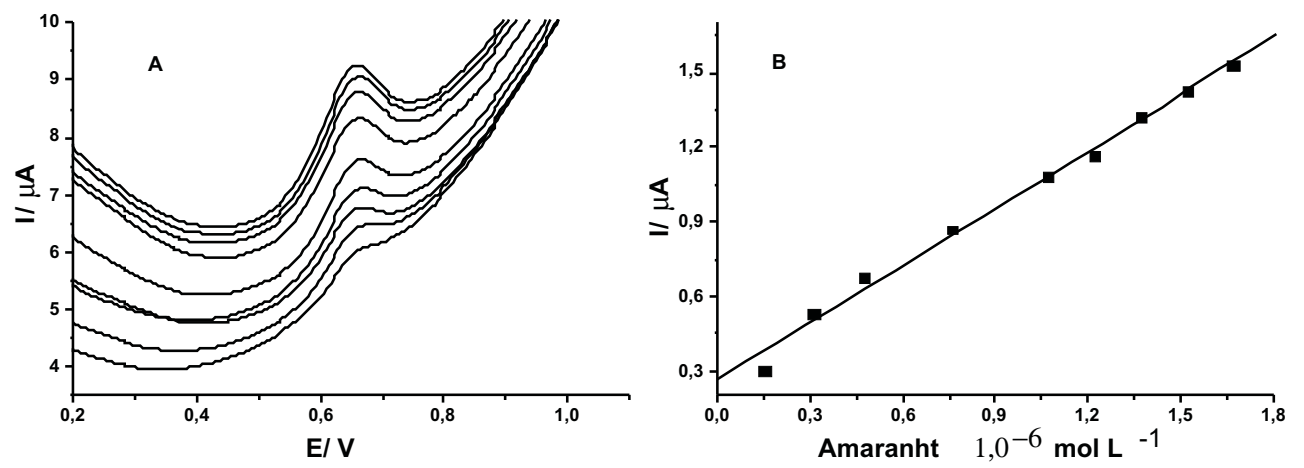

Figura 9. Voltamperogramas de adsorción A) y curva de calibrado B) de amaranth; pH: 2,$0 ; \mathrm{C}_{\mathrm{CPB}}: 10,0 \times 10^{-6} \mathrm{~mol} \mathrm{~L}^{-1} ; \mathrm{t}_{\text {ads }}: 90 \mathrm{~s} ; \mathrm{E}_{\text {ads }} 0,40 \mathrm{~V}$; otros parámetros iguales a figura 4.

\section{Validación y estudio de interferencias.}

La falta de estándares certificados no permitió su validación. Por otro lado, se dopó una muestra de bebida gaseosa con 4,0 x $10^{-4} \mathrm{molL}^{-1}$ de amaranth. Cuantificando por el método de adición de estándar $3,7 \times 10^{-4} \mathrm{molL}^{-1}$ con un error relativo de $-7,5 \%$. otros colorantes, como sudán I, sudán II y tartracina no interfieren con la señal del amaranth.

\section{Análisis de muestras reales:}

Se analizó bebidas gaseosas de colores rojo y algunas gelatinas del mismo color, pero no se encontró amaranth.

\section{CONCLUSIONES}

El método propuesto es sencillo, de bajo costo y es conveniente para determinar amaranth en matrices bebidas gaseosas y alimentos que contienen colorantes como gelatinas, etc. Otros colorantes no interfieren. La falta de estándares certificados no permite su total validación.

\section{AGRADECIMIENTOS}

Los autores agradecen a FONDECYT por el financiamiento del Proyecto Postdoctorado 3120030. A la Vicerrectoría de Investigación de la Universidad de la Amazonía, Colombia, por el proyecto de semillero 2014. 


\section{BIBLIOGRAFÍA}

1. M. Gómez, V. Arancibia, C. Rojas, E. Nagles. International Journal of electrochemical science. 2012; 7: 7493-7502

2. A. Arango, L.F. Garcés. Revista Lasallista de investigación. 2009; 6(2):32-38

3. M. Salazar-Gastélum, E. Reynoso-Soto, S. Lin, S. Perez-Sicairos, R. Félix-Navarro. Journal of Environmental Protection. 2013; (4) 137-143

4. N. Pourreza, S. Elhami. Journal of the Iran chemical society. 2009; 6(4): 784-788

5. M. Perez-Urquiza, J.L. Beltran. Journal of Chromatography. 2000; 898: 271-275

6. M. Maa, X. Luoa, B. Chenb, S. Su, S. Yao. Journal of Chromatography A, 2006; 1103: 170-176.

7. H. Abu Shawish, N. Abu Ghalwa, S. Saadeh, H. Harazeen. Food Chemistry. 2013; 138: 126-132

8. W. Mei L., Z. Jing, D. Ni N., Z. Xiao L., C. Zhi D. Journal of AOAC International. 2013; 96: 625 .

9. P. Wang, X. Hu, Q. Cheng, X. Zhao, X. Fu, K. Wu. Journal of Agriculture Food Chemistry. 2010; 58(23): 12112-12116

10. S. Chandran, L. A. Lonappan, D.Thomas, T. Jos, K. G. Kumar. Food Analytical Methods. 2014; 7: 741-746

11. Q. Han, X. Wang, Z. Yang,W. Zhu, X. Zhou, H. Jiang. Talanta. 2014; 123: 101-108

12. M. Panduranga, E. Niranjana, B.E. Kumara, B.S. Sherigara, K. Vasantakumar. International Journal of electrochemical science. 2008; 3: 588-596

13. M. G. Panelli, A. Voulgaropoulos, Electroanalysis, 1993; 5(5-6): 355-373

14. J.C. Miller, J.N. Miller, Estadística y Quimiometría para Química Analítica, cuarte edición, ADDISON-WESLEY, 124-125, 2002. 